\title{
PRIVACIÓN DE LIBERTAD EN LOS ESTABLECIMIENTOS DE MEDELLÍN ${ }^{3}$
}

\author{
DEPRIVATION OF LIBERTY IN PENITENTIARIES IN MEDELLIN
}

Recibido: Junio de 2011 - Revisado: Febrero de 2012 - Aceptado: 30 de Mayo de 2012

\author{
Por: Juan David Posada Segura ${ }^{1}$ \\ Luz Marina Acevedo Jaramillo ${ }^{2}$
}

\section{RESUMEN:}

La investigación "Estado de los derechos fundamentales de las personas privadas de la libertad en los establecimientos penitenciarios y carcelarios de Medellin" se ocupó de definir el contexto histórico y actual de los establecimientos de privación de la libertad en el municipio de Medellín. De acuerdo con ello se describen los establecimientos que han existido en el municipio y los que actualmente existen para la privación de la libertad de miembros de la Policía Nacional, el Ejército Nacional y civiles adultos hombres y mujeres. Como una manera de evidenciar que la privación de la libertad es discriminada en diversos tipos de establecimientos y que aquellos que se construyeron como la solución a grandísimos problemas penitenciarios y carcelarios simplemente entran a hacer parte del problema.

\section{PALABRAS CLAVE:}

Cárceles, penitenciarías, Medellín, privación de la libertad.

\begin{abstract}
:
The research "the state of the fundamental rights of people deprived of liberty in correctional and prison institutions in Medellin" dealt with defining the historical and the current context of institutions of deprivation of liberty in the municipality of Medellin. Based on that, those that have existed in the municipality are described, as well as those which currently exist for the deprivation of liberty for the members of the National Police, the National Army, and civilian adult men and women. As a way to demonstrate that the deprivation of liberty is discriminated in various types of those institutions, and that those which were built as the solution to huge prison and penitentiary problems, are simply part of the problem.
\end{abstract}

\section{KEY WORDS:}

Prisons, Penitentiaries, Medellín, Deprivation of Liberty.

\footnotetext{
${ }^{1}$ Investigador principal. Docente e Investigador de la Facultad de Derecho y Ciencias Politicas de la Universidad de Antioquia-Colombia. Doctor Cum Laude en Derecho Sociología Jurídico-Penal (Universidad de Barcelona), Magister en Derechos Humanos (Universidad Internacional de Andalucía) y Abogado (Universidad de Antioquia) Colombia. juandavid@posadasegura.org www.posadasegura.org

${ }^{2}$ Coinvestigadora. Abogada Unidad permanente para los Derechos Humanos (Convenio Personería de Medellín -Secretaría de Gobierno) Colombia. Especialista en Gestión Pública, Diplomada en género y políticas públicas, Diplomada en justicia restaurativa, Diplomada en sistema de gestión de calidad, Conciliadora en derecho y Abogada. lmacevedo@une.net.co.
} 


\section{Introducción.}

La idea de desarrollar esta investigación surgió después de haber realizado un ejercicio similar en el año 2009, en el que miembros del Grupo en Derecho Penitenciario, acompañaron el proceso de diagnóstico en materia de Derechos Humanos realizado por la Personería de Medellín; en aquel año, se realizaron encuestas a las personas privadas de la libertad en el establecimiento de mediana seguridad y carcelario de Medellín, popularmente conocido como Bellavista, a la entonces reclusión de mujeres de Medellín, conocida popularmente como el Buen Pastor y a la cárcel de la Policía Nacional, ubicada en el barrio Robledo Aures. Fue así, que tras aquel ejercicio se vio la necesidad de realizar una investigación en la que se profundizara en los orígenes de la privación de la libertad en Medellín y en las características básicas de estos establecimientos.

El mundo de la privación de la libertad es un mundo que presenta velos que sólo pueden ser retirados en la medida en que se aproxime más y más a él, esto hace que la percepción de esa realidad sea siempre diferente, aunque no por ello errada. Teniendo lo anterior como punto de partida se consideró sumamente importante, antes de entrar a definir en una segunda fase, la opinión de las personas privadas de la libertad en Medellín sobre la situación de sus derechos humanos o fundamentales, definir cuáles son y han sido los establecimientos utilizados para la privación de la libertad en el municipio de Medellín.

La violación de los derechos humanos de las personas privadas de la libertad no es un hecho poco diagnosticado, por el contrario un observador desprevenido podría concluir fácilmente que se trata de un hecho sobre-diagnosticado. Sin embargo la descripción histórica penitenciaria y carcelaria no es uno de los temas que logremos encontrar con facilidad, ya que las historias penitenciarias suelen quedar opacadas por las gravísimas realidades actuales y de todos los tiempos.

La reciente creación de un nuevo complejo penitenciario y carcelario en la ciudad de Medellín, en el cual se han presentado huelgas de hambre y amotinamientos en pocos meses por parte de las personas privadas de la libertad, que en éste se encuentran, constituye una situación coyuntural que obliga a la realización de un diagnóstico directo como el que se realizó para aportar en la obtención de un mayor y mejor conocimiento de la realidad penitenciaria y carcelaria de la ciudad y así contribuir a la labor que desarrolla la Personería de Medellín y otros organismos en la solución de los problemas que se detecten.

Sin embargo, esa situación de denuncias de violación de derechos humanos en este nuevo establecimiento es una constante que ha ocupado a todos los establecimientos para la reclusión de hombres y mujeres civiles que existen y han existido en el municipio de Medellín y seguramente en otras latitudes; independientemente de las razones que llevaron a su creación, del modelo arquitectónico utilizado o incluso del contexto social y político en que se han inaugurado tales centros. De acuerdo con lo anterior en esta primera fase de la investigación quiere evidenciarse la estructural existencia de problemas que han tenido los establecimientos de privación de la libertad, teniendo como excusa a los de Medellín, para extraer conclusiones que son aplicables a la estructura penitenciaria y carcelaria en general.

En la prisión existen multitud de dimensiones, todas ellas dignas de ser establecidas como objeto de estudio e investigación; sin embargo, teniendo siempre como eje los derechos fundamentales, en las dos fases de esta investigación, se prestó atención a tres aspectos pilares que tradicionalmente caracterizan el sistema penitenciario: 
De un lado, la frecuente opacidad, falta de transparencia $y / u$ ocultismo, que algunos administradores penitenciarios o algunas personas privadas de la libertad que cuentan con poder, pueden imponer sobre el interior de las penitenciarias y cárceles, a pesar de que éstas sean instituciones públicas de las que los ciudadanos han de estar informados.

En segundo lugar, el hecho de que la prisión (por sus necesidades disciplinarias) se constituya como un espacio de dominación en cual son muy frecuentes las situaciones tanto de indefensión como de vulneración de los derechos fundamentales de los allí recluidos e incluso del cuerpo de custodia y vigilancia. Situaciones éstas que ocurren a pesar de tratarse de un contexto establecido por el propio sistema penal, por tanto debería prevalecer el principio de legalidad y los demás principios del sistema penal en general.

En tercer lugar, el carácter de irreparable, las consecuencias físicas, psicológicas y sociales que el hecho de estar privado de la libertad trae consigo para los detenidos, para sus familias y para los funcionarios del cuerpo de custodia.

Estas preocupaciones desarrolladas en la investigación se inscriben dentro de la perspectiva de la Sociología del Control Penal (Bartta, 1986). Este marco epistemológico implica, entre otros asuntos, tener muy presente las cuestiones de definición del delito y los procesos de selectividad del delincuente, teniendo conciencia de que el delito es una entidad construida legalmente y no es un ente natural, y la determinación acerca de quién es delincuente, está drásticamente afectada por estereotipos y prejuicios.

En el marco de la mencionada disciplina (conocida como Sociología del Control Penal) se han realizado trabajos investigativos, en los que partiendo de la observación directa de los centros penitenciarios, se determina la situación material de los derechos fundamentales de las personas en ellos recluidas. La mayoría de ejemplos, de este tipo de investigaciones se encuentran en trabajos españoles, los cuales deben ser tenidos en cuenta, no para copiar de manera inconsulta los modelos en ellas implementados por los investigadores, sino para evidenciar, a partir del conocimiento de elementos comunes el hecho de que la privación de la libertad presenta características similares independientemente de las condiciones económicas o socio-culturales de los países donde ésta se ejecute. ${ }^{4}$

Sin embargo, también existe ésta clase de investigaciones en el plano nacional: Enen primer lugar ha de citarse la labor de la Oficina en Colombia del Alto Comisionado para los Derechos Humanos y de la Defensoría del Pueblo, quienes periódicamente publican informes sobre el estado de los derechos fundamentales en los centros de reclusión colombianos; de igual manera ha de considerarse muy especialmente la labor hecha por la Corte Constitucional Colombiana que, de cara a la producción de la Sentencia T-153 de 1998 realizó un importante trabajo que dio cuenta del estado de los derechos fundamentales de las personas detenidas al interior de los centros penitenciarios del país, y allí, como era de esperarse, se estudió con especial cuidado la situación de la cárcel Bellavista. El estudio se ocupó de los derechos fundamentales en general $\mathrm{y}$, de algunos en particular concentrándose en los centros penitenciarios de mayor capacidad en términos de reclusión de personas. También ha de tenerse en cuenta la investigación desarrollada por la Procuraduría General de la Nación y la Universidad de San Buenaventura en la que se verificó la condición de infraestructura de todos los establecimientos de Antioquia "Estado de los centros y lugares de reclusión de Antioquia" (Vallejo \& Posada, 2011). 
En el plano local, también existen estudios sobre La privación de la libertad en la ciudad de Medellin; en ellos debe destacarse especialmente el trabajo que permanentemente desarrolla la Oficina Delegada para los Derechos Humanos de la Personería de Medellín, la cual semestralmente emite informes sobre la situación de los derechos humanos y fundamentales en la ciudad (en general) y en los establecimientos de privación de la libertad en la ciudad de Medellín (en particular), ocupándose de los casos que requieren una atención inmediata, tales como: problemas con la alimentación, el servicio de salud, las actividades de trabajo, estudio y enseñanza, la sobrepoblación penitenciaria conocida como hacinamiento y las demás graves violaciones a los derechos de los privados de libertad en los establecimientos penitenciarios y carcelarios de la ciudad de Medellín, esto es, el establecimiento Penitenciario de Mediana Seguridad y Carcelario de Medellín, conocido popularmente como Bellavista, y el nuevo Complejo Penitenciario y Carcelario de Medellín conocido como "El pedregal".

A manera de conclusión sobre el "estado del arte" en materia de estudio sobre la situación de los derechos fundamentales de las personas privadas de la libertad en Medellín, puede afirmarse que existe buena cantidad de informes en el plano sociológico, sin embargo no son comunes las investigaciones en las que se dé cuenta de los establecimientos penitenciarios y carcelarios desde un punto de vista histórico o de contexto general actual.

De acuerdo con lo anterior, el objetivo de esta primera fase de la investigación fue rastrear históricamente los más importantes establecimientos para la privación de la libertad de los hombres y mujeres en de Medellín, para lo cual resulta necesario documentar el contexto de la privación de la libertad de adultos hombres y mujeres en el municipio de Medellín asi como describir el tipo de establecimientos que se han utilizado para la privación de la libertad en Medellin.

\section{Metodologia.}

La investigación se desarrolló en un espacio físico delimitado que corresponde a los Establecimientos Penitenciarios y Carcelarios de Medellin: ${ }^{5}$ Establecimiento Penitenciario de Mediana Seguridad y Carcelario de Medellín, Complejo Penitenciario y Carcelario de Medellín, ${ }^{6}$ centro de reclusión de la Policía Nacional y centros de reclusión militar.

Los elementos que se constituyeron como objeto de observación, en esta primera fase, fueron: el estado de los establecimientos, las características de la población que allí se encuentra o encontró y el nivel de garantía de los derechos humanos de acuerdo con informes de autoridades de control o medios de comunicación.

La diversidad de elementos variables y factores que participan en una investigación como la desarrollada requirierón, desde el punto de vista metodológico, la combinación de diferentes procedimientos asi como las técnicas desarrolladas en función de los objetivos, pero también de las circunstancias particulares que envuelven el objeto de estudio. Se ejecutó un tipo de investigación socio-jurídica donde se concertaron elementos normativos-sociales, de manera que, primero se visitaron los establecimientos de reclusión con el fin de recolectar la información necesaria para ésta primera fase de la investigación, y ya que la investigación inmediatamente continuaría en su segunda fase, se pasó a un segundo momento que fue la aplicación de las encuestas a las personas privadas de la libertad, posteriormente se realizó la interpretación-sistematización del material encontrado, con el fin de elaborar un informe que contuviera los resultados y conclusiones pertinentes. Asi, en este artículo se presentan 
los resultados de esta primera fase y se espera que en uno posterior se presenten los resultados de la aplicación de encuestas. Por lo tanto, utilizamos la combinación de estrategias de investigación cuantitativa y cualitativa.

Con la intención de explicar las relaciones existentes entre un hecho con su contexto, la investigación planteó un método hermenéutico de textos - contextos, información oficial y resultados del trabajo de campo, es decir, se parte de unos datos presuntamente reales para verificar la realidad de los mismos mediante la realización de entrevistas a funcionarios, exinternos, etc. asi como encuestas a las personas privadas de la libertad.

Como instrumento principal se tuvo el formato de encuesta, entendida la encuesta como una manera de dialogar con la persona

, sin olvidar que se trata de una herramienta bastante limitada, pero que abre una puerta a la experiencia del otro.

"La encuesta (mediante cuestionario estructurado) impone ciertamente una fuerte rigidez al diálogo, pero en cambio tiene la ventaja de mantener un enorme número de "encuentros conversacionales" con personas muy diversas; además, en cada uno de estos diálogos, se trata sobre los mismos asuntos, lo que hace fácilmente comparable entre sí y, por la estructura muy formalizada que presentan, se pueden obtener en un período de tiempo muy breve" (Rios \& Cabrera, 1998).

\section{Historia de la privación de libertad en el municipio de Medellín}

En este apartado se referenciarán brevemente algunos aspectos generales de tres establecimientos de privación de la libertad que hemos tenido en Medellín, como fueron: la recientemente cerrada reclusión de mujeres de Medellin, (conocida popularmente como el Buen Pastor), la cárcel del distrito judicial de Medellín (conocida popularmente como la Ladera) y la Casa cárcel de Medellín.

\section{Reclusión de Mujeres de Medellin 1894-2010}

La Reclusión de Mujeres de Medellín fue fundada en 1894 mediante Ordenanza No. 5 del 29 de mayo de ese año con el fin de crear un sitio adecuado para ubicar a las mujeres "detenidas o sentenciadas por asunto criminal o de policia” (Roldan, J. C., Gonzalez, L. \& Arquidiocesis de Medellin, 2005).

Esta creación se dio de la mano de la comunidad religiosa del Buen Pastor, congregación que promueve el bienestar y defiende los derechos de las personas, en especial de la mujer en vulnerabilidad y riesgo social (trabajadoras sexuales, desplazadas y en extrema pobreza) y sus familias, a partir del desarrollo de líneas de intervención y promoción social (capacitación y formación) en su contexto personal, familiar y social, que favorezcan su crecimiento integral y su transformación social, emanados de un proceso de evangelización permanente.

La reclusión abrió sus puertas, previo contrato firmado por el alcalde municipal Dionisio Arango, su primera directora fue la Hermana María del Sagrado Corazón, una religiosa de la comunidad, por solicitud del entonces Monseñor Joaquín Pardo, Obispo de la ciudad de Medellín. 
Las necesidades que llevaron a fundar la cárcel fuerón las de mejorar la suerte de las internas atendiendo mejor la parte moral y alimenticia de los establecimientos de castigo, ya que sólo existía una cárcel donde eran recluidos tanto hombres como mujeres. Empezó a funcionar en el antiguo puente de las Pizas con 60 internas y religiosas extranjeras.

En enero en 1903 se trasladó a la Casa de Pichincha donde se encuentran actualmente las torres de Bomboná; alli se hizo la clasificación de condenados y sindicados. El 24 de septiembre de 1912 se abrió la sección gran clase de voluntarias de niñas de jóvenes que necesitaban ayuda donde funcionaba la cárcel (San Javier la América) hasta el mes de Julio del año 2010.

El 20 de enero de 1916, por ordenanza 37 del 1 de mayo, se fundó La Escuela Departamental con veinte niños menores, cuyas clases eran recibidas en sus mismos centros. En marzo de 1929 se abrió un centro para niñas dificiles y de baja conducta y a partir del 24 de abril de 1961 se empezó a recibir niñas por inspección de menores (INPEC, 2011).

En el año 2003, el Tribunal de Antioquia ordenó desalojar al Instituto Nacional Penitenciario y Carcelario (INPEC) las instalaciones donde se hallaba la reclusión "Buen Pastor", Carrera 95 \# 42 C 02 (San Javier -La América) por tres años de atraso en el pago de los cánones de arrendamiento que se adeudaban a las religiosas de la comunidad. Según datos suministrados por medios de prensa como El Colombiano, la deuda ascendía a 363 millones de pesos.

Sin embargo, argumentando que Colombia tiene más de cuarenta años de atraso en su sistema penitenciario y carcelario, el Ministerio del Interior y de Justicia se propuso modernizar la infraestructura de las cárceles del país, garantizar la resocialización a través de un nuevo enfoque de la política penitenciaria y disminuir el índice de hacinamiento que llegó al cuarenta y dos por ciento y que representa un sobrecupo de veintiocho mil personas en todo el país. La estrategia a nivel nacional contempló la construcción de diez establecimientos que conjugaran en su arquitectura los conceptos de seguridad y derechos humanos, cuyos diseños tuvieron el apoyo del Bureau de Prisiones de Estados Unidos (El Colombiano, 2011). Y para el caso de la ciudad de Medellín, además contaron con instalaciones de propiedad del INPEC.

La reclusión el Buen Pastor, según datos suministrados por la página web oficial del Inpec, contaba con 480 internas mujeres bajo la dirección de la Señora Orfa Nelly Henao Giraldo ${ }^{7}$ en el año 2010. En el mes de julio de ese mismo año el establecimiento cierra sus puertas e inician los traslados de las internas al nuevo establecimiento ubicado en el Corregimiento San Cristóbal de la ciudad de Medellín.

Este establecimiento albergaba también el único anexo psiquiátrico de la ciudad de Medellín en el que se albergaba a hombres con trastorno mental sobreviniente, esto es, hombres que no fueron declarados inimputables por trastorno mental en el proceso pero su condición hacía necesario alojarlos en unas instalaciones especiales. Dichas instalaciones desaparecieron con el cierre de este anexo psiquiátrico, con lo que, ahora en Antioquia no existe un lugar para éste tipo de personas con trastorno mental, quedando sólo un pequeño lugar que no tiene las condiciones necesarias y que está ubicado en la zona de sanidad en el establecimiento popularmente conocido como bellavista. 
Al momento en que se cierra este establecimiento, era un edificio vetusto con enormes dificultades para garantizar en él el desarrollo de actividades de tratamiento penitenciario para las mujeres alli recluidas.

Cárcel distrital de Medellín “la ladera” 1921-1976 (López, 2004).

El terreno en el que se construiría la "Ladera", al parecer para el siglo XIX era un campo apto para el pastoreo; era poca la población que alli se asentaba. Paulatinamente, a principios del siglo XX este sector fue poblado por inmigrantes de otras partes del departamento, que llegaban a Medellin en búsqueda de oportunidades, incluso por los propios medellinenses excluidos del centro de la ciudad, quienes veían allí un lugar de asentamiento. En 1914 esta propiedad fue comprada por el Gobernador Julián Cock Bayer por $\$ 12.000 .00$ pesos.

La "Casa de La Ladera", desde fines del siglo XIX, fue objeto de interés para distintos fines por parte de funcionarios y dirigentes regionales, hasta que finalmente se le dio la función que había recomendado el señor Cock Bayer, pero no para reclusión de mujeres, pues ya para finales de la década de 1910 y principios de la de 1920 se ventiló con mayor vehemencia la propuesta de una nueva cárcel distrital de varones para Medellín.

Para la construcción de la cárcel del Distrito Judicial de Medellín se hizo cargo el Arquitecto Ingeniero del Departamento, el belga Agustín Goovaerts, cumpliendo con las cláusulas de su contrato con el departamento de Antioquia, celebrado en 1920. Para el efecto adaptó y acondicionó la ya referida antigua Casa de La Ladera, y adyacente a ésta comenzó a construir pabellones o patios con celdas para la detención de individuos. Es de aclarar que, en un principio ésta fue pensada para cárcel, es decir, estaba siendo construida para detenidos preventivamente y sumariados, no para condenados.

Durante el proceso de construcción de la cárcel objeto de estudio, en la década de 1920, aquella institución fue conocida con el nombre de Cárcel Celular de Varones de Medellín. Posteriormente el adjetivo "celular" fue abandonándose ${ }^{8}$ y reemplazándose por el de judicial, pasando a ser llamada Cárcel Judicial de Varones, y al cabo de los años, simplemente "La Ladera”.

La cárcel distrital de Medellín "La Ladera", comenzó a construirse en 1921 en propiedad del departamento con dineros nacionales y departamentales, en el barrio Enciso de dicha ciudad. Comenzó a funcionar dos años después, en noviembre de 1923, cuando el edificio se hallaba aún inconcluso. El primer director de la Cárcel distrital de Medellín "La ladera", fue el Señor Julio Viana.

Dos años después de haber puesto en funcionamiento una parte de la cárcel, y al ver que los trabajos para concluirla no se adelantaban satisfactoriamente, algunos funcionarios y la prensa local empezaron a preocuparse y a llamar la atención sobre este hecho a las autoridades competentes. En tal sentido se consideraba como un error el traslado de los presos a la nueva cárcel celular. La Cárcel de Varones de Medellín, desde los inicios de su construcción en 1921, empezó a ser considerada una de las joyas arquitectónicas de la ciudad. Por esos años, los presos de La Ladera participaron en la construcción del Estadio Atanasio Girardot. Precisamente, uno de sus reclusos más famosos fue el escritor nadaísta Gonzalo Arango, quien pasó tres días alli (El Mundo, 2008, 18 de abril). 
Con el pasar del tiempo, el crecimiento de la población reclusa y el abandono estatal para la terminación de la obra, acentuaron problemas tales como la inseguridad, pues se presentaron fugas que no dejaron de ser sensacionales; el hacinamiento, que llegó a superar el 400\%, pues para la época de su clausura, en enero de 1976, albergaba poco menos de 3400 presos, siendo su capacidad para 800 presos mal acomodados, y la indisciplina interna, ya que fueron constantes las riñas, heridas y muertes de presos dentro del plantel. Situaciones que se mantuvieron a lo largo de la existencia de la cárcel.

Una vez más lo que se construyó para solucionar problemas penitenciarios de la época terminó cerrando en medio de un enorme problema que simplemente se trasladó a otro lugar del que se escribirá más adelante "bellavista". Hoy y tras varios años de abandono, éste lugar pasó a ser el lote para el parque biblioteca del mismo nombre.

\section{Casa cárcel de Medellín.}

El sistema de reclusión especial para incursos en delitos culposos derivados de incidentes de tránsito, denominado "Casa Cárcel del Conductor" fue instituido a instancia de las peticiones que en su época hicieron las organizaciones sindicales del sector, lideradas por el Sindicato Nacional de Choferes de Colombia, hoy Sindicato Nacional del Transporte de Colombia, "Sindinal", para que se reformara el régimen penitenciario y se clasificara a los reclusos de acuerdo al tipo de delito, pues, según ellos, no era lógico que un conductor sindicado por homicidio y/o lesiones culposas en accidente de tránsito recibiera el mismo tratamiento carcelario de personas acusadas de delitos dolosos, se les confinara en un mismo sitio de reclusión con grave peligro para su integridad personal y se les obligara a compartir con toda clase de delincuentes.

Estas peticiones fueron respaldadas por un gran movimiento gremial del transporte que incluyó protestas diversas, huelgas, paros, mítines y pronunciamientos continuos que se produjeron a principios de la década de los años 60 en el siglo pasado siglo.

Finalmente, el gobierno aceptó la validez de los argumentos sociales y jurídicos de los representantes gremiales de los conductores. Por tal razón la Dirección General de Prisiones, hoy Inpec, dictó la Resolución 0335 de 1966, reconociendo la necesidad de adoptar un sistema especial de reclusión para los conductores sindicados de delitos culposos derivados de accidentes de tránsito y autorizó al Sindicato Nacional del Transporte de Colombia, la creación, organización y administración de las casas cárceles de conductores, con el aporte económico exclusivo de las organizaciones Sindicales y como único apoyo estatal el suministro de los respectivos guardias de custodia.

A partir de este momento, se inició la creación de las casas cárceles, primero en la ciudad de Bogotá, luego en Medellín, Cali, Manizales, Cúcuta, Bucaramanga, Villavicencio, Duitama, Armenia, Pereira, Barrancabermeja y otras ciudades, hasta llegar a un número de 14, todas con el aporte económico de las organizaciones sindicales.

Hay que subrayar que el servicio de casa cárcel podía ser prestado por entidades privadas, pero la dirección y vigilancia seguía a cargo de la dirección general de prisiones, ya que la casa cárcel debía estar anexa a un establecimiento de reclusión nacional cercano. En la ciudad de Medellín, la casa cárcel funcionó en el sindicato de choferes ubicado en el sector de las Torres de Bombona. ${ }^{9}$ 
En 1975, el Gobierno Nacional reglamentó su funcionamiento a través del decreto 1419, en el cual se ratificó su existencia y funcionamiento así como la calidad de administradores y aportantes de las organizaciones sindicales, debido a que se consideraba un servicio de carácter social y su comercialización presentaría situaciones incongruentes con su objeto, función y características en las que se consideró su calidad de servicio social.

En diciembre 11 de 1991 se dictó el Decreto 2758 del Ministerio de Justicia, el cual se alejó del criterio inicial de servicio social a cargo de las organizaciones sindicales, de reivindicación gremial y establecimientos carcelarios, cuya naturaleza reñía con la explotación comercial al autorizara cualquier particular a crear y organizar las casas cárceles para conductores. Sin embargo, en razón a que no existía forma de comercialización y explotación mercantil, las casas cárceles siguieron funcionando bajo la administración de las organizaciones sindicales.

La Resolución No 035/91 autorizó la creación de la sección para mujeres en las casas cárceles de conductores, habida cuenta del aumento de la población femenina que ejercía la actividad de conducir vehículos y la comisión eventual de delitos culposos en su ejercicio. La Ley 65 de 1993, Código Penitenciario y Carcelario, ratificó la existencia de las casas cárceles de conductores.

Finalmente, el Inpec, a través de su Consejo Directivo dictó el Acuerdo 010 de 1997 que modificó todo el criterio histórico de estos centros especiales de reclusión y abrió la puerta para su comercialización generalizada, con grave desmedro para los conductores y usuarios del servicio, para el control del Estado y para la concepción social y sindical de estos especiales centros de reclusión, ya que para los conductores profesionales el accidente de tránsito, antes que un delito culposo es un accidente de trabajo.

A partir de la ley 769 de 2002, se empieza a hablar de Centros integrales de atención (CIA) como los establecimientos habilitados por el ministerio de trasporte, en los que se presta el servicio de escuela y de casa-cárcel para la rehabilitación de los infractores de las normas de tránsito, pero es la Resolución 005868 del 21 diciembre de 2010, la que habilita al Centro Integral de Atención al Infractor de Tránsito.

Finalmente encontramos en la prensa local un artículo publicado el 28 de julio de 2011, donde se informa que el Ministerio de Transporte habilita a la firma Ciatran S.A, para prestar servicios como centro integral de atención para infractores de tránsito en Medellín y que se iniciará este año la construcción de una casa-cárcel para la cuidad (El Colombiano, 2011). Sin embargo, al momento de cierre de este informe no se cuenta con información oficial de su existencia, por lo que se mantiene la información de que se trata de un tipo de centro de reclusión desaparecido en la historia de la ciudad.

\section{Breve descripción de los actuales establecimientos de privación de libertad en Medellín, para miembros de la policia nacional, el ejército nacional.}

Si bien, la investigación que ahora se presenta, sólo se ocupará en su segunda fase de los establecimientos de privación de libertad de Medellín destinados a adultos civiles, lo que excluye establecimientos para la privación de libertad de miembros de la Policía Nacional y del Ejercito Naciona $1^{10}$ ubicados en la ciudad de Medellín, estos se describirán brevemente para significar que también son establecimientos en los que se encuentran personas privadas 
de la libertad en Medellín y para que el lector tenga un panorama más completo del sistema de privación de la libertad que opera en el municipio, aunque no se trata de establecimientos de los que comúnmente se tenga información, muy probablemente porque no son establecimientos en los que suelan presentarse situaciones de violación de derechos humanos.

\section{Centro de Rehabilitación Aures}

El Centro de Rehabilitación Aures, es el establecimiento carcelario de la Policía Nacional, es decir, se trata de un establecimiento del orden nacional oficialmente utilizado para la detención preventiva de funcionarios de la Policía Nacional que estén siendo investigados por actos del servicio o por delitos señalados en el Código Penal Militar; lo que obviamente implica que allí nunca son privadas de la libertad personas que no tengan relación alguna con este organismo policial. Se trata del único establecimiento de privación de la libertad con que cuenta la Policía Nacional en el Municipio de Medellín, está ubicado en el barrio Robledo Aures, calle $79 \mathrm{~N}^{\circ} 96 \mathrm{~B}-260$. Tiene una capacidad instalada para 150 personas.

El personal de establecimiento está conformado por funcionarios administrativos que son miembros de la Policía Nacional en servicio activo, de igual manera que los funcionarios de custodia que son miembros activos de la policía nacional que se ocupan de la vigilancia externa del establecimiento.

Los servicios de salud son prestados a los privados de la libertad a través del Hospital Pablo Tobón Uribe, tanto para medicina general como para urgencias. Sin embargo debe tenerse en cuenta que el área metropolitana de Medellín cuenta con un hospital de la policía nacional, lo que implica que allí se prestarán todos los servicios especiales de salud; en todo caso dentro del establecimiento cuentan con una enfermeria para la prestación de servicios básicos de salud.

La alimentación es suministrada por el Inpec, pero es manipulada por los mismos privados de la libertad, ellos son los encargados del manejo y almacenamiento. Los servicios de educación son ofrecidos por el SENA, lo que facilita que el establecimiento cuente con programas de formación técnica que les permiten redimir pena por esa actividad. El establecimiento tiene sitios para el esparcimiento en los cuales los privados de la libertad pueden realizar actividades educativas, culturales, religiosas, entre otras.

En este establecimiento cuentan con opciones laborales como los proyectos productivos, la granja, los talleres, el rancho, entre otros, que les permiten redimir pena y en algunos casos recibir una remuneración.

Los pabellones son llamados módulos en este establecimiento, cuenta con 2 módulos; a nivel de infraestructura el establecimiento se encuentra en muy buen estado, lo que hace que las celdas, los talleres, aulas y demás espacios dedicados a las actividades de los privados de la libertad resulten plenamente ajustadas a lo que exigen las Reglas Mínimas para el Tratamiento de los Reclusos y que resulten compatibles con las exigencias constitucionales y legales de garantía de los derechos de los seres humanos, alli privados de la libertad y faciliten de paso la realización de actividades de tratamiento penitenciario. 
El ambiente general del establecimiento es tranquilo, lo que muy probablemente se ve influenciado por factores como la disciplina y línea de mando que se respeta entre los funcionarios y exfuncionarios de la policía que se encuentran alli privados de la libertad y por las condiciones de infraestructura, sumada a la tranquilidad que ofrece la excelente vista que sobre la ciudad entrega este lugar.

\section{Actuales establecimientos para la privación de la libertad de militares ubicados en Medellin.}

Seguidamente se mencionarán en este informe tres centros utilizados para la privación de la libertad de funcionarios del Ejército Nacional que se encuentren en proceso de investigación por delitos relacionados con el servicio o por delitos señalados en el Código Penal Militar. Deberá tenerse en cuenta que se trata de pequeños centros de reclusión ubicados dentro de instalaciones militares de Medellín y que en el caso del Área Metropolitana de Medellín, el principal centro de reclusión militar se encuentra en el Batallón Pedro Nel Ospina Ubicado en el Municipio de Bello por lo que no será objeto de este trabajo en razón de la delimitación territorial.

\section{Centro de reclusión del Batallón Atanasio Girardot}

El centro de reclusión del Batallón de Infantería No. 10 Atanasio Girardot, pertenece al Ejercito Nacional, es decir, es un establecimiento del orden nacional. Está ubicado en el Municipio de Medellín, Barrio Villa Hermosa, Carrera 66E No.39 - 84; en razón de que se trata de una pequeña instalación que hace parte del Batallón, el responsable de éste es el mismo comandante del batallón que actualmente es el Teniente Coronel Antonio José Dangon Gulzat.

El personal del establecimiento está conformado por miembros del Ejército Nacional en servicio activo. Ya que como se mencionó en éste y en los demás casos de "salas de reflexión militares", se trata de pequeñas instalaciones en las que se recluye a miembros o exmiembros de las fuerzas militares, dentro de los batallones, por lo que son administrados por miembros activos de la misma unidad militar y utilizan toda la infraestructura de estos batallones, así los servicios de salud son prestados en el establecimiento, el cual cuenta con una enfermería, una sala de recuperación y una farmacia. Los alimentos son los mismos que se ofrecen al personal del batallón y se entregan en los mismos horarios, de acuerdo con el menú preestablecido.

El SENA ofrece servicios de educación técnica a los militares y exmilitares privados de la libertad pueden redimir pena por esa actividad. De igual forma cuentan con diferentes actividades de trabajo, como una oportunidad para invertir su tiempo libre y a la vez obtener una redención de pena.

El batallón cuenta con lugares de esparcimiento que les permiten realizar actividades culturales y religiosas también a los privados de la libertad.

El centro de reclusiones una pequeña edificación separada de los alojamientos y oficinas ubicada a pocos metros del puesto de control de ingreso del Batallón. Cuenta con un pabellón para todos sus internos, lo que significa una capacidad instalada para 20 personas. Su infraestructura está en buen estado, cuentan con servicios públicos básicos y tal como se mencionó antes con las instalaciones del batallón son utilizadas para actividades de los privados de la libertad. 


\section{Sala de reflexión del Batallón Pedro Justo Berrio}

La sala de reflexión del batallón Pedro Justo Berrío Infantería 32, hace parte del Ejército Nacional, lo que significa que es un establecimiento del orden nacional; está ubicado en el Municipio de Medellín, barrio Belén las playas al costado del Aeropuerto Olaya Herrera en la Carrera $70 \mathrm{~N}^{\circ}$ 1-50; el actual comandante de este batallón y consecuentemente el responsable de este centro de reclusión es el Teniente Coronel Edgar Alberto Rico Pulido.1 igual que en las demás salas de reflexión, la administración de este pequeño establecimiento con capacidad instalada para 18 personas, está a cargo de los mismos uniformados del batallón y la seguridad perimetral es asumida como un turno más de centinela por los soldados asignados al batallón.

Los servicios de salud son prestados al interior del establecimiento, el cual cuenta con una enfermería, un dispensario y un área de hospitalización. Los alimentos son proporcionados por el Batallón, por lo tanto allí mismo se realiza la manipulación y el almacenamiento como corresponde a todas las unidades militares.

En cuanto a la educación, cuentan con servicios del SENA que presta programas de formación técnica, permitiendo redimir pena por realizar esta actividad. Así mismo, tiene diversas opciones de actividades laborales para ser realizada por sus internos. Para invertir el tiempo libre cuenta con lugares para realizar actividades culturales y lúdicas.

Posee dos celdas comunes para sus internos, su infraestructura está en buenas condiciones y tiene los servicios públicos básicos. Se trata de dos habitaciones ubicadas en la parte posterior del batallón, con lo que se encuentran aislados de los alojamientos y oficinas administrativas.

\section{El centro de Reclusión del batallón Jorge Eduardo Sánchez.}

El centro de Reclusión del Batallón de Artillería N 4, Jorge Eduardo Sánchez, más conocido como el BAJES, hace parte del Ejército Nacional, lo que significa que es un establecimiento del orden nacional; está ubicado en el Municipio de Medellín, bario Buenos Aires en la calle 45 $\mathrm{N}^{\circ}$ 18-85; actualmente es comandado por el Teniente Coronel Luis Fernando Delgado Romero.

El establecimiento tiene una capacidad instalada para unas 24 personas, cuenta con funcionarios administrativos que hacen parte del personal del Ejército Nacional, al igual que en los demás batallones son rotados de acuerdo con las necesidades del servicio y los custodios son los soldados que se encuentren en turno de centinela.

Los servicios de salud son prestados a sus internos al interior del reclusorio, tiene un dispensario para ello y cuenta con personal médico y zona de hospitalización. El servicio de alimentos pertenece al mismo batallón.

En cuanto a los servicios de educación no cuenta con programas especiales, sin embargo cuenta con la oportunidad de realizar actividades de trabajo para ser desarrolladas al interior del establecimiento, pudiendo redimir pena por realizar esas actividades. Para invertir el tiempo libre cuentan con actividades culturales y religiosas. El establecimiento cuenta con un solo pabellón, tiene los servicios públicos básicos y su infraestructura está en buenas condiciones. 


\section{Actuales establecimientos para la privación de la libertad en Medellín para adultos civiles}

Este apartado se ocupará de los dos grandes establecimientos para la privación de la libertad de Medellín: de un lado el establecimiento conocido como Bellavista, que si bien no queda geográficamente en Medellín, si recibe la denominación "Establecimiento Penitenciario de Mediana Seguridad y Carcelario de Medellín" EPMSC, y son la Alcaldía de Medellín y la Personería de esta ciudad, las que mantienen un constante trabajo en este establecimiento, ya que el municipio de Bello tras cerrar su cárcel municipal se limitó a celebrar un convenio con este establecimiento para que reciba allí a los privados de la libertad del municipio y a la Personería de Bello sólo realiza puntuales actividades de seguimiento a casos concretos pero no actividades globales de seguimiento a las condiciones de privación de la libertad que allí se desarrollan.

Por otra parte este apartado se ocupará del establecimiento de privación de la libertad denominado COPED o complejo penitenciario y carcelario el Pedregal, que es el más reciente establecimiento de la ciudad y se ocupa de la privación de la libertad de hombres y mujeres en Medellin.

\section{Establecimiento penitenciario de mediana seguridad y carcelario de Medellín (epmscmed) "bellavista"}

\section{Historia}

La construcción de la Cárcel Nacional Bellavista inició en 1971, como un nuevo espacio para trasladar a los presos de la cárcel La Ladera, que tal como se anotó antes, dejó de funcionar en enero de 1976 cuando se trasladaron los 3400 privados de la libertad en carros de la basura del Municipio, escoltados por la fuerza pública (El Colombiano, 2011). De acuerdo con ello, Bellavista fue el establecimiento construido para solucionar los problemas de hacinamiento y en general de violaciones de derechos que entonces tenía la Ladera.

El nuevo establecimiento quedó ubicado en el Municipio de Bello, sector Machado, entre los barrios las Vegas y la Camila. Fue construido para albergar 1500 personas, quienes serian condenados, aquellas personas no tuvieran que cumplir penas mayores a cinco años, y aunque muy posteriormente se realizó una ampliación de 300 cupos, el uso excesivo de la detención preventiva, obligó a que se albergaran tanto a sindicados como a condenados, y el aumento de los tipos penales, la disminución de beneficios penitenciarios y otras causas relacionadas con la implementación del nuevo sistema de tendencia acusatoria hicieron que llegara a superar las 7400 personas privadas de la libertad en épocas críticas recientes.

La inauguración de la cárcel sólo se pudo realizar el 14 de junio de 1977, encontrándose a cargo del penal el Director Luis Octavio Valencia. Desde su inicio, los internos se mostraron descontentos por la calidad del reclusorio y desde finales de la década de los ochenta las quejas sobre insalubridad y hacinamiento fueron continuas (El Colombiano, 2011).

Además, se vivía un ambiente tenso, hasta el punto de ser llamada "la cárcel más peligrosa de Latinoamérica". Las fugas y las muertes violentas dentro de la prisión eran comunes. Aunque existieron fugas masivas, como la del 18 de agosto de 1987, cuando 17 presos salieron del penal en un furgón, la más recordada fue la fuga de diciembre de 1988, cuando un helicóptero aterrizó en el patio dos del penal y se llevó a cinco presos. 
Para 1990 el promedio de muertes dentro del penal era de cuatro a la semana, superando en el doble a los tiempos más críticos que había tenido la Ladera. En julio de 1994 fue inaugurado el Pabellón de Máxima Seguridad de la cárcel, lo que añadió una capacidad de 300 cupos al establecimiento, alcanzando así una capacidad real de 1800 cupos, lo que difiere sustancialmente de los 2424 cupos con los que cuenta oficialmente (El Tiempo, 2004 20 de Marzo).

Para el año 2001, el Alto Comisionado de las Naciones Unidas para los Derechos Humanos Oficina en Colombia realizó un informe de la situación carcelaria en Colombia, teniendo como referente la sentencia T-153 de 1998. En éste informe se refirió a la situación del EPMSC Bellavista de Medellín de la siguiente forma: "En el caso de la cárcel de Distrito Judicial de Bogotá-La Modelo y la cárcel de Distrito Judicial de Medellín-Bellavista, la Misión realizó inspecciones similares a las efectuadas por la Corte Constitucional en 1998, incluyendo una visita nocturna al último centro. La Misión constató el empeoramiento de las condiciones documentadas en la sentencia T-153 de abril de 1998 de la Corte Constitucional, que halló "un estado inconstitucional de cosas" y ordenó, entre otras cosas, la descongestión de todos los centros penitenciarios y carcelarios y la separación completa de los internos sindicados y condenados, en un plazo máximo de cuatro años. Ninguna de estas disposiciones de la sentencia de la Corte Constitucional se ha cumplido, si bien se ha dado cumplimiento a otras".

En el informe del año 2008, se hace referencia al hacinamiento, falta de ventilación e iluminación que presenta el EPMSC Bellavista. En cuanto a la atención en salud de los internos, se advierte: "En su gran mayoría, las áreas de sanidad no reúnen las condiciones de higiene, asepsia y ventilación que requiere un sitio destinado a la prestación del servicio de salud. Con ello no sólo se viola el derecho a la salud de los reclusos sino también su derecho a la dignidad" (Personería de Medellín, 2008).

\section{Descripción actual.}

El establecimiento penitenciario de Mediana Seguridad y Carcelario de Medellín (EPMSCMED), hace parte de la Regional Noroeste del Inpec, lo que significa que es un establecimiento del orden nacional. Está ubicado en el Departamento de Antioquia, Municipio de Bello, en la Diagonal $44 \mathrm{~N}^{\circ} 39-145$.

Aunque este establecimiento no queda en Medellín, sino en Bello, su denominación lo convierte en establecimiento de la ciudad, además es en el que se materializan los convenios que tiene el municipio de Medellín con el Inpec para la detención preventiva de este municipio, que no cuenta con establecimiento del orden municipal.

El personal del establecimiento está conformado por funcionarios del nivel administrativo, por guardias de seguridad adscritos al Inpec, por bachilleres que se encuentran prestando su servicio militar obligatorio y por el personal de tratamiento conformado por educadores, psicólogos, y trabajadores sociales, entre otros.

Los servicios de salud han prestados en los últimos años por intermedio de CAPRECOM, no sólo para la asistencia médica, sino para el servicio de urgencias. Al interior del establecimiento cuentan con una enfermeria, una farmacia y una sala de observación para tratar enfermedades comunes. Así mismo tiene un lugar en el que, sin ser anexo psiquiátrico, 
se recluye a los pacientes que requieren de esta clase de atención. Este puede ser en los últimos tiempos quizás el más visible de los gravísimos problemas que en materia de derechos humanos presenta este centro de reclusión, ya que se han presentado casos de muerte por inasistencia médica.

Los servicios de alimentación son prestados a través de un contrato con un ente privado que se encarga de suministrar los alimentos, por su parte la manipulación se hace al interior del establecimiento.

En cuanto a la educación los internos tienen oportunidades de realizar programas educativos de educación formal tales como básica primaria y básica secundaria; así mismo programas informales dentro de los que resaltan manualidades, artes y oficios varios. En este establecimiento se cuentan como opciones laborales los proyectos productivos como la granja y gaviones, talleres de calzado, confecciones, talla de cuero, brigadas de limpieza, entre otros, permitiendo así que puedan redimir pena por realizar alguna de esas actividades. Adicionalmente se cuenta con espacios para la recreación y el esparcimiento, tiene una cancha múltiple, espacios al aire libre en cada patio, lugares para la realización de los talleres.

Al interior de este centro de reclusión al momento de la visita, encontramos nueve pabellones, los cuales no llevan un número consecutivo debido a las modificaciones que se han realizado al interior del establecimiento, debido a la gran demanda de población reclusa.

Los servicios públicos básicos se encuentran en mal estado, al igual que la infraestructura general de todo el centro de reclusión, que fácilmente podría colapsar. Así una vez más el que en su momento se presentó como la solución a los problemas de la Ladera, es fuertemente criticado por las degradantes condiciones a las que somete a los allí privados de la libertad, es por ello que la ciudad una vez más pensó en que la solución era contar con un nuevo establecimiento, tal como se verá en el apartado siguiente.

\section{Complejo penitenciario y carcelario de Medellin "el Pedregal"}

\section{Historia.}

Bajo la Resolución 2668 del 5 de Marzo de 2010 se creó el establecimiento carcelario de Medellín y se integró al mismo la reclusión de mujeres de Medellín, más tarde, en la Resolución 1282 del 8 de abril de 2011 se adoptó la denominación de complejo carcelario y penitenciario dentro de la clasificación de los establecimientos de reclusión del orden nacional, y finalmente mediante la Resolución 1797 del 5 de Mayo de 2011 se crea un complejo carcelario y penitenciario, (INPEC, 2011) el cual se conoce hoy día como El Pedregal.

El Complejo Carcelario y penitenciario de Medellín - Pedregal- está ubicada en lo que se conocía como finca La Teresita, kilometro seis antigua vía al mar, en el corregimiento San Cristóbal de la ciudad, abrió sus puertas en el mes de Julio del año 2010 cuando se dieron los primeros traslados de las internas provenientes de la reclusión de mujeres del Buen Pastor.

Antes de su construcción, ésta fue la casa de paz, donde se adelantaban negociaciones entre el Gobierno Nacional y el ELN en cabeza de Gerardo Bermúdez, alias "Francisco Galán" (Caracol Radio, 2006, 19 de agosto) 
Este establecimiento surgió como medida del Gobierno Nacional en la construcción de nuevas cárceles producto de su política criminal y penitenciaria, con una inversión de 115 mil millones de pesos, un área de 52.000 metros cuadrados y una capacidad de 2.754 internos, se construyó de ésta manera la denominada "cárcel 5 estrellas".

Los traslados empezaron a darse el 12 de Julio del año 2010 a las 10:30 de la mañana, iniciaron con la llegada de tres buses de servicios especiales ocupados por 98 reclusas y en medio de un gran dispositivo de seguridad en el que participaron funcionarios del Instituto Nacional Penitenciario y Carcelario, efectivos motorizados de la Policía y del Ejército Nacional. Ya en la tarde, se realizó un operativo mayor, con el desplazamiento de 150 internas para completar un número de 248 reclusas que ocupaban las nuevas instalaciones carcelarias y penitenciarias. Los traslados continuaron hasta el 14 de Julio del mismo año de manera paulatina.

El establecimiento, actualmente cuenta con una capacidad para albergar 1252 mujeres y 1129 hombres; funciona a su vez como establecimiento carcelario de hombres y reclusión de mujeres, divididos por complejos, por ello se encuentran alli recluidos ambos géneros.

En sus orígenes, se trató de dos establecimientos: la reclusión de mujeres de Medellín a la que se trasladaron las de la desaparecida reclusión conocida como "El Buen Pastor" que ya no era una edificación apta y el establecimiento carcelario de Medellín, al cual fueron enviados los internos en situación de sindicados del establecimiento penitenciario y carcelario de Medellín- Bellavista, con el ánimo de contribuir con la disminución del hacinamiento del lugar y procurar con ello dar cumplimiento a la normatividad en materia penitenciaria que prohíbe albergar en un mismo sitio a sujetos cuya situación jurídica es de condenados con los sindicados, además fue un compromiso adquirido por el Inpec frente al Municipio de Medellín, quien donó el predio bajo esa condición. Ello significaba que para cada establecimiento debía operar una administración independiente, por tanto al año siguiente de su constitución, se opta por unificar ambos establecimientos y enmarcarlos bajo el concepto de Complejo carcelario y penitenciario, así se tendría una sola dirección y por ende una sola administración del complejo en general.

\section{Descripción actual.}

El Complejo Penitenciario y Carcelario El Pedregal, hace parte de la Regional Noroeste del INPEC, lo que significa que es un establecimiento del orden nacional; El personal del establecimiento está conformados por personal del nivel administrativo, además de los guardias adscritos al INPEC, siendo hombres los encargados del ala masculina y mujeres, las encargadas de custodiar La femenina. Adicionalmente en el ala masculina cuentan con bachilleres que están prestando el servicio militar. El personal de tratamiento está conformado por instructores, trabajadoras sociales, psicólogos, médicos, enfermeros, odontólogos, entre otros.

Tanto para hombres como para mujeres, el servicio de salud es uno de los más cuestionados, ya que son numerosas las denuncias por inasistencia médica general y especializada, por no suministro de medicamentos o no realización de exámenes ordenados por los médicos, etc. lo que sumado a los enormes problemas que ha evidenciado el prestador de este servicio hacen que la existencia de un área de enfermería, una sala de recuperación, cuarto de insumos y consultorio médico, resulten ser uno más de los casos en los que la existencia de 
infraestructura, sin soporte de recurso humano hagan inexistente o de mala calidad a un servicio que implica la materialización de uno de los más importantes derechos humanos o fundamentales.

Los servicios de alimentación tanto para hombres como para mujeres son contratados con un ente privado que proporciona los alimentos, los cuales son manejados al interior del establecimiento. El servicio de alimentación es compartido entre hombres y mujeres.

Tanto hombres como mujeres cuentan con programas de educación formal e informal, siendo la alfabetización y la educación básica primaria las más realizadas. Así mismo, las mujeres participan en programas de artes y cultura. Además cuentan con espacios para el esparcimiento como canchas y aulas para desarrollar las actividades. Los hombres y mujeres pueden laborar en diversas actividades para redimir su pena. Los hombres se especializan en talleres, proyectos productivos, brigadas de limpieza, y como instructores de educación. Las mujeres trabajan más que todo en limpieza y en el expendio. En cuanto a los pabellones, hay seis para hombres y nueve, para mujeres, Todos estos espacios cuentan con servicios públicos básicos en condiciones regulares, además la iluminación es escasa debido a que no entra el sol a los pabellones de hombres. Su infraestructura a nivel general está en buenas condiciones aunque evidencia un deterioro muy importante en el poco tiempo que tiene de funcionamiento.

Este establecimiento que se construyó con la idea de ayudar a superar los problemas de hacinamiento de "bellavista" y la necesidad de trasladar a las mujeres del Buen Pastor, hoy es objeto de importantes denuncias por violación de derechos humanos y si bien se ha mantenido al margen de las graves condiciones de hacinamiento de otros centros, ya alcanzó su capacidad instalada y ha hecho que se piense una vez más que en Medellín es necesaria la construcción de otros establecimiento para la privación de la libertad.

\section{Conclusiones}

La privación de la libertad en Medellín no es de un solo tipo y no aplican las mismas observaciones para los diversos tipos de establecimientos, así la privación de la libertad que se aplica a miembros de la policía nacional y de las Fuerzas militares, evidentemente cumple con las básicas condiciones que ordenan las reglas Mínimas para el Tratamiento de los reclusos; en estos establecimientos no se presentan problemas de hacinamiento generador de violación de todo tipo de derechos humanos, el estilo de lo que la Corte constitucional denominó como estado de cosas inconstitucional en su famosa sentencia t 153 de 1998; por el contrario estos son lugares de respeto por los derechos fundamentales de los privados de la libertad.

Lo mismo sucedió en su momento con la privación de la libertad para las personas involucradas en incidentes de tránsito, ya que ésta tampoco fue nunca objeto de señalamientos por violación de derechos humanos.

No puede afirmarse lo mismo de los establecimientos dedicados a la privación de la libertad de hombres y de mujeres civiles en la ciudad, incluso resulta necesario aclarar que estos deben ser tratados también de manera diferenciada; así, mientras la privación de la libertad de las mujeres en el centros de reclusión conocido como el buen pastor presentaba denuncias por violación de puntuales derechos humanos sin llegar nunca a la condición de un estado de 
cosas inconstitucional, lo que se evidenciaba en la cantidad de acciones de tutela presentadas; se vio fuertemente incrementado al ser trasladadas al nuevo centro de reclusión, así pasamos de un promedio de privación de la libertad de mujeres en Medellín de quinientas personas a "llenar" un establecimiento de 1200 en apenas dos años, cuando esa no era la población promedio.

En el caso de los hombres no adscritos a las fuerzas armadas, la historia es sustancialmente más delicada, ya que la historia de construcción de establecimiento en la ciudad y las razones para ello han sido las mismas y las respuestas parece ser que seguirán siendo iguales. "La Ladera" se construyó para hacer frente a las gravísimas condiciones que ofrecía la privación de la libertad en la ciudad de Medellín, así que se construyó "Bellavista" para dar solución a esos problemas y hoy es quizás emblema de violación de derechos en el país siendo uno de los más hacinados centros de reclusión del orden nacional y apareciendo en todos los informes que sobre violación de derechos humanos de las personas privadas de la libertad se emiten en el país; fue así que se pensó en construir "el Pedregal" cuando bellavista tenía un promedio de población de cinco mil personas, lo que se aliviaría con los aproximadamente mil doscientos cupos que abriria la ciudad para hombres; sin embargo no sólo se llenaron esos cupos sino que la misma bellavista creció unos dos mil quinientos cupos en promedio; pero a pesar de que vemos estos problemas la idea que recientemente surgió para solucionar una vez más los gravísimos problemas de bellavista es la promesa de construcción de un nuevo mega-centro de reclusión en el que se albergue a unos dos mil hombres.

La separación entre detenidos preventivos y condenados nunca ha logrado ser una realidad en nuestros centros de reclusión, razones de orden práctico lo han impedido, así cada que se ha construido un establecimiento se ha señalado expresamente que constituyen una oportunidad para separar a los detenidos preventivos de los condenados, sin embargo a pocos dias de ser inaugurados esa directriz se ha convertido en letra muerta y la separación por razones de situación jurídica se ha convertido en una mera declaración normativa que ha querido superar el concepto "Establecimiento Penitenciario y Carcelario". No es que se crea que ésta es la solución a los graves problemas de violación de derechos humanos de los privados de la libertad, pero si ha de tenerse en cuenta que es un factor que ayuda en la agudización del problema.

Espacios que ya habiamos conquistado como la separación de hombres y mujeres en los centros de reclusión se ven matizados ahora por el naciente concepto "Complejo Penitenciario y Carcelario" que hace oficialmente un único establecimiento de lo que deberían ser dos plenamente separados; una vez más razones de carácter económico y administrativo resultan consideradas.

Todo lo anterior significa que en este punto en concreto no hemos aprendido de la local historia de la privación de la libertad, para comprender que estos fenómenos, que se repiten en el país y en América Latina en general, tienen soluciones en otras esferas que permitieran llevar el derecho penal y su ejecutor, el derecho penitenciario, a su mínima expresión; a que el derecho penal realmente se usara como última solución posible. 


\section{Resferencias Bibliográficas}

Acevedo, L. M. (2009). Historia Invisibilizada. Revista Holística Jurídica, $N^{\circ} 6$. Universidad de San Buenaventura. Medellín. p.p. 21-30.

Amaya, C. E. (2001). El drama de las cárceles en Colombia. Bogotá: Ediciones librería del profesional.

Baratta, A. (1986). Criminología crítica y crítica del Derecho Penal. Introducción a la Sociologia jurídico-penal. Siglo XXI, México.

Bernal, C. E. (2004). ¿Cárceles de verdad o cárceles del terror?. Círculo de investigación "Castigo y sociedad". Actualidad colombiana. Edición N 380.

Castaño, O. (2011). Cementerio de libertades. Segunda edición. Medellín: Editorial Prográficas.

Centro de Investigaciones En Política Criminal. (2007). Análisis de las políticas públicas en torno a la prisión. Bogotá: Universidad Externado de Colombia.

Chacón, I. (2005). Contexto penitenciario colombiano. Bogotá: Ediciones jurídicas Andrés Morales.

Corporación Colectivo de Abogados Jose Alvear Restrepo. (2000). La sin razón, Situación carcelaria en Colombia. Bogotá: Rodríguez quito editores.

Defensoría Del Pueblo Colombia. (2004). Resolución Defensorial Nº 30.

Defensoria Del Pueblo Regional Antioquia. (2011). Informe Defensorial sobre la situación penitenciaria y carcelaria en los establecimientos carcelarios del Área Metropolitana de Medellín: Reclusión de Mujeres, Establecimiento Penitenciario y Carcelario de Medellín "BELLAVISTA", Establecimiento Carcelario de Medellin "EL PEDREGAL" Y Establecimiento Penitenciario de Mediana y Alta Seguridad de Itagüí.

Fundación Comité De Solidaridad Con Los Presos Políticos. (2003). Tortura en tiempos de "seguridad democrática". Bogotá.

Gaitán, O. L. (2000). Análisis de la situación carcelaria en Colombia: un enfoque cualitativo. CIJUS. ediciones Uniandes.

Gómez, R. (2000). Bellavista ¿ mito o realidad? Siglo 3000. Medellín.

Llerena, I. (2010). Avances y primeros informes del Observatorio de Derechos Humanos del EPMSC de Medellín. III Simposio Internacional Penitenciario y de Derechos Humanos, Universidad de San Buenaventura, Medellín, p.p. 197-202.

López, O. L. (2003). Sistema progresivo penitenciario alternativo, Un modelo de intervención para el sistema penitenciario colombiano. INER. Medellin. Universidad de Antioquia. 
López, J. (2004). Cárcel distrital de Medellín "La Ladera". 1921-1976. Trabajo de Grado para optar al título de Historiador. Universidad de Antioquia. Facultad de Ciencias Sociales y Humanas. Medellín.

Martínez, D. M. (2000). Situación carcelaria en Colombia. Conferencia regional sobre la situación carcelaria en la Región Andina. Quito: INREDH.

Oficina De Asistencia Técnica Legislativa. Senado de la República. (2004). Estudio de antecedentes sobre el sistema penitenciario y carcelario. Gaceta del Congreso año XIII No 8. Bogotá.

Orrego, J. J. (2001). El drama humano en las cárceles. La realidad del sistema penitenciario y carcelario colombiano. Medellín.

Personería De Medellín. (2008). Informe Ejecutivo de Derechos Humanos.

Personería De Medellín. (2009). Informe Ejecutivo de Derechos Humanos.

Personería De Medellín. (2010). Informe elaborado por la abogada Luz Marina Acevedo Jaramillo. Convenio Personeria de Medellín-Secretaria de Gobierno.

Personería De Medellín. (2011). Informe sobre la situación de Derechos Humanos en Medellín.

Posada, J. D. (2009). Sistema penitenciario. Estudio sobre la privación de la libertad y los derechos fundamentales relacionados. Medellín: Comlibros.

Posada, J. D. (2010). Derechos de los reclusos en los pronunciamientos judiciales, revista estudios de derecho, año LXVII, segunda época, número 150, p.p. 205-227.

Reyes, J. F. (1999). Jurisprudencia constitucional y derechos de las personas privadas de la libertad. En: Nuevo Foro Penal, Nº 61, Medellín, p.p. 179-220.

Rivera, I. (1992). Cárcel y derechos humanos. Un enfoque relativo a la defensa de los derechos fundamentales de los reclusos. Barcelona: J. M. Bosch editor.

Rivera, I. (1997). La devaluación de los derechos fundamentales de los reclusos. La construcción jurídica de un ciudadano de segunda categoría. Barcelona: J.M. Bosch editor.

Rivera, I. (2006). La cuestión carcelaria. Historia, epistemología, derecho y política penitenciaria. Buenos Aires: Editores del puerto.

Roldan, J. C., González, L. \& Arquidiócesis De Medellín. (2005). Situación de los derechos humanos en las cárceles del área metropolitana. Reclusión de mujeres Buen Pastor -Medellín.: Pastoral Social caritas Arquidocesanas.

Vallejo, C. P. \& Posada, J. D. (2011). Privación de la libertad en Antioquia. Revista Rostros y Rastros. Instituto de Estudios del Ministerio Público. Bogotá. Año 3. №6. p. 46-56. 


\section{Cibergrafia}

Boletin De Prensa No 86. (2012, julio 26). Extraído el 28 de julio, 2012, En:

http: / / www.personeriamedellin.gov.co/index.php/Sala-dePrensa/Boletines / Preocupacion-por-la-atencion-en-salud-que-se-brinda-a-lapoblacion-carcelaria-de-la-ciudad.html

Boletín De Prensa No. 201. (2010, 27 de octubre). Extraído el 28 de octubre, 2010, En: http:/ / www.personeriamedellin.gov.co/index.php/Sala-de-Prensa/Boletines/LaPersoneria-de-Medellin-insta-al-gobierno-nacional-a-tomar-las-medidasnecesarias-para-el-restablecimiento-de-los-derechos-de-los-reclusos-delEstablecimiento-Carcelario-el-Pedregal.html

Caracol Radio. (2011, 1 de junio). Inpec no es fuente de financiación de Caprecom: Vargas Lleras. Extraído el 1 de junio, 2011, EN:

http://www.caracol.com.co/noticias / actualidad/inpec-no-es-fuente-definanciacion-de-caprecom-vargas-lleras/20110601/nota/1482469.aspx

Caracol Radio. (2006, 19 de agosto). Se traslada la 'casa de paz' del ELN a Envigado (Antioquia). Extraído el 07 de mayo, 2011, En:

http://www.caracol.com.co/nota.aspx?id=322246

Casa Editorial El Tiempo. Extraído el 15 febrero, 2011, En:

http: / / ads.casaeditorialeltiempo.com/colombia/antioquia/condiciones-en-lacarcel-el-pedregal-de-medellin_8055100-1.

INPEC. (2011). Establecimiento-Epmsc de Medellín. Extraído el 15 de mayo, 2011, En: http://www.inpec.gov.co/portal/page/portal/Inpec/SeccionInpeccomoinstitucion/ EstablecimientosPenitenciarios / DetalleEstablecimiento?establecimiento $=964$.

INPEC. (2011). Directores y Subdirectores Establecimientos de Reclusión. Extraído el 03 de abril, 2011, En:

http://www.inpec.gov.co/portal/page/portal/Inpec/SeccionInpeccomoinstitucion/ EstablecimientosPenitenciarios/Establecimientos?regional=4

INPEC. (2011). Extraído el 08 de agosto, 2011, En:

http://www.inpec.gov.co/portal/page/portal/INPEC_CONTENIDO/NOTICIAS\%20 Y\%20NORMATIVIDAD/MARQUESINA1/Tab/R001797_05052011.pdf

Los derechos de las personas privadas de su libertad. Extraido el 08 de julio, 2011, EN: http://www.cidh.org/countryrep/colom99sp/capitulo-14.htm

Metrosalud. (2012, 19 de julio). Metrosalud prestará servicios de salud a población carcelaria. Extraído el 19 de julio, 2012, En: http://www.metrosalud.gov.co/inter/joomla/index.php/noticias/372-metrosaludprestara-servicios-de-salud-a-poblacion-carcelaria- 
Oficina De Las Naciones Unidas Contra La Droga Y E1 Delito. (2007). Recopilación de reglas y normas de las Naciones Unidas en la esfera de la prevención del delito y la justicia penal. Extraído el 10 de julio, 2012, EN:

http://www.unodc.org/pdf/criminal_justice/Compendium_UN_Standards_and_No rms_CP_and_CJ_Spanish.pdf

Periódico El Colombiano. (2011) El hacinamiento ahonda el problema en Bellavista. Extraído el 15 de Mayo, 2011, En:

http://www.elcolombiano.com/BancoConocimiento/H/hacinamiento_ahonda_el_p roblema/hacinamiento_ahonda_el_problema.asp

Periodico El Colombiano. (2011). Comenzó traslado de reclusas del Buen Pastor a la nueva cárcel de San Cristóbal. Extraído el 03 de abril de 2011, En: http:/ /www.elcolombiano.com/BancoConocimiento/C/comenzo_traslado_de_reclu sas_del_buen_pastor_a_la_nueva_carcel_de_san_cristobal/comenzo_traslado_de_re clusas_del_buen_pastor_a_la_nueva_carcel_de_san_cristobal.asp

Periódico El Colombiano. (2011). Medellín tendrá una casa cárcel. Extraído el 28 de julio, 2011, En:

http://www.elcolombiano.com/BancoConocimiento/M/medellin_tendra_una_casa _carcel/medellin_tendra_una_casa_carcel.asp

Periodico El Colombiano. (2012, 05 de mayo). La salud de los presos está en la sala de urgencias. Extraído el 07 de mayo, 2012, En:

http://www.elcolombiano.com/BancoConocimiento/L/la_salud_de_los_presos_est a_en_la_sala_de_urgencias/la_salud_de_los_presos_esta_en_la_sala_de_urgencias

Periodico El Tiempo. (1994). Minjusticia visita pabellón de seguridad. Extraído el 20 de marzo, 2012, En: http://www.eltiempo.com/archivo/documento/MAM-185178

Periódico E1 Tiempo. (2011, 21 de junio). Atención en salud de los presos no será exclusividad de EPS Caprecom. Extraído el 17 de agosto, 2011, En:

http: / / www.eltiempo.com/justicia/ARTICULO-WEB-NEW_NOTA_INTERIOR9691526.html

Periodico El Tiempo. (2012, 16 de julio). Se prepara decreto para reformar la atención de salud en las cárceles. Extraído el 16 de julio, 2011, En:

http: / / www.eltiempo.com/vida-de-hoy / salud / ARTICULO-WEBNEW_NOTA_INTERIOR-12036030.html

Periodico El Mundo. (2011, 18 de Abril). El señor de las 40 cenas. Extraído el 15 de julio, 2011, En: http://www.elmundo.com/portal/resultados/detalles/?idx=176054

RCN. (2011, 22 de enero). Problemas en la cárcel de "El pedregal" en Medellín. Extraído el 14 de Marzo, 2011, En:

http: / / www.rcnradio.com/noticias / 22-01-11/problemas-en-la-c-rcel-de-elpedregal-en-medell-n 
Notas

${ }^{3}$ El presente artículo es resultado final de la primera fase de la investigación: "Estado de los derechos fundamentales de las personas privadas de la libertad en los establecimientos penitenciarios y carcelarios de Medellin", desarrollada por la Oficina Permanente de Derechos Humanos de la Personería de Medellín y el grupo de investigación Sistema Penitenciario de la Universidad de Antioquia. Todo el equipo de auxiliares fue conformado por estudiantes de la Facultad de Derecho y Ciencias Politicas de la Universidad de Antioquia siendo miembros activos del Semillero de Derecho Penitenciario, (www.derechopenitenciario.org) que es un grupo para la formación de investigadores en materia penitenciaria avalado por el grupo de investigación Sistema Penitenciario. Alexandra Fernández Rojas; Ana Lucia Restrepo Castro; Carolina Garcia Ortiz; Geraldine Cartagena Garcia; Jorge Luis Arango Mesa; y Leidy Johana Dávila Cano.

${ }^{4}$ Investigación realizada por el Observatorio de Sistema Penal y Derechos Humanos (OSPDH) de la Universidad de Barcelona titulada "Condicions de l'empresonament a Catalunya"; ésta se realizó en 11 centros penitenciarios catalanes: Centre Penitenciari d'homes de Barcelona 4 ("La Model"), Centre Obert de Barcelona, Centre Penitenciari de Dones de Barcelona ("Wad-Ras"), Centre Penitenciari de Joves de Barcelona ("La Trinitat"), Centre Penitenciari de Brians, Centre Penitenciari de Quatre Camins 6, Centre Penitenciari de Tarragona, Centre Penitenciari de Ponent, Centre Obert de Lleida, Centre Penitenciari de Girona 7, Centre Penitenciari de Figueres. De igual manera resulta pertinente la investigación realizada por la Asociación Pro Derechos Humanos, denominado Informe sobre la situación de las prisiones en España, publicado por Editorial fundamentos Colección Ciencia en Madrid, en 1999.Haciendo uso de la encuesta a los detenidos, como método para la obtención de información la Universidad Pontificia Comillas realizó también un trabajo de investigación sobre la situación de las personas al interior de las penitenciarias en España en 1998 y en 2000 publicado por RÍOS MARTÍN, Julián Carlos y CABRERA CABRERA, Pedro José, Mil voces presas, Universidad Pontificia Comillas, Madrid, 1998. y Mirando el abismo, el régimen cerrado, Universidad Pontificia Comillas, Madrid, 2000.

${ }^{5}$ Debe tenerse en cuenta que aunque este establecimiento conocido como Bellavista realmente está ubicado en el municipio de Bello, su nombre (EPMSC Medellín) lo convierte en establecimiento de Medellín.

${ }^{6}$ Se trata del nuevo establecimiento del orden nacional para hombres y para mujeres ubicado en el corregimiento de San Cristóbal del municipio de Medellín.

${ }^{7} \mathrm{Al}$ momento de realización de este informe se desempeña como diputada de la Asamblea departamental de Antioquia.

${ }^{8}$ Seguramente porque le resultaba imposible cumplir con la característica indispensable de la reclusión en celda individual que contempla el sistema penitenciario celular.

${ }^{9}$ En relación a este "Sindicato de Choferes", nunca se logró conseguir información de su parte, porque su personal aducía no tener información alguna sobre el servicio que allí se prestó hace algunos años. Así que la información fue suministrada por el entonces Personero de Medellín, Jairo Herrán Vargas.

${ }^{10}$ En la ciudad de Medellín no existen establecimientos para la privación de la libertad de miembros de la Fuerza Aérea o de la Armada Nacional. 
